University of Nebraska - Lincoln

DigitalCommons@University of Nebraska - Lincoln

\title{
A-MAC: Adaptive Medium Access Control for Next Generation Wireless Terminals
}

Mehmet C. Vuran

Georgia Institute of Technology, mcvuran@cse.unl.edu

Ian F. Akyildiz

Georgia Institute of Technology

Follow this and additional works at: https://digitalcommons.unl.edu/csearticles

Part of the Computer Sciences Commons

Vuran, Mehmet C. and Akyildiz, Ian F., "A-MAC: Adaptive Medium Access Control for Next Generation Wireless Terminals" (2007). CSE Journal Articles. 90.

https://digitalcommons.unl.edu/csearticles/90

This Article is brought to you for free and open access by the Computer Science and Engineering, Department of at DigitalCommons@University of Nebraska - Lincoln. It has been accepted for inclusion in CSE Journal Articles by an authorized administrator of DigitalCommons@University of Nebraska - Lincoln. 


\title{
A-MAC: Adaptive Medium Access Control for Next Generation Wireless Terminals
}

\author{
Mehmet C. Vuran, Member, IEEE, and Ian F. Akyildiz, Fellow, IEEE
}

\begin{abstract}
Next Generation (NG) wireless networks are envisioned to provide high bandwidth to mobile users via bandwidth aggregation over heterogeneous wireless architectures. NG wireless networks, however, impose challenges due to their architectural heterogeneity in terms of different access schemes, resource allocation techniques as well as diverse quality of service requirements. These heterogeneities must be captured and handled dynamically as mobile terminals roam between different wireless architectures. However, to address these challenges, the existing proposals require either a significant modification in the network structure and in base stations or a completely new architecture, which lead to integration problems in terms of implementation costs, scalability and backward compatibility. Thus, the integration of the existing medium access schemes, e.g., CSMA, TDMA and CDMA, dictates an adaptive and seamless medium access control (MAC) layer that can achieve high network utilization and meet diverse Quality of Service $(\mathrm{QoS})$ requirements.

In this paper, an adaptive medium access control (A-MAC) layer is proposed to address the heterogeneities posed by the NG wireless networks. A-MAC introduces a two-layered MAC framework that accomplishes the adaptivity to both architectural heterogeneities and diverse $Q 0 S$ requirements. A novel virtual cube concept is introduced as a unified metric to model heterogeneous access schemes and capture their behavior. Based on the Virtual Cube concept, A-MAC provides architecture-independent decision and QoS based scheduling algorithms for efficient multi-network access. A-MAC performs seamless medium access to multiple networks without requiring any additional modifications in the existing network structures. It is shown via extensive simulations that A-MAC provides adaptivity to the heterogeneities in NG wireless networks and achieves high performance.
\end{abstract}

Index Terms-Adaptive medium access control, heterogeneous networks, heterogeneous $\mathrm{QoS}$ requirements, next generation wireless networks, virtual cube concept.

\section{INTRODUCTION}

$\mathbf{N}$ EXT GENERATION (NG) wireless networks are expected to provide mobile users with a freedom of roaming between diverse set of wireless architectures as shown in Fig. 1. Since an NG wireless terminal will operate in these various types of networks, the Medium Access Control (MAC) layer will encounter different protocols that are already deployed in the access points (AP) of these networks. The various types

Manuscript received May 12, 2004; revised March 2, 2005, and January 27, 2006; approved by IEEE/ACM TRANSACTIONS ON NETWORKING Editor M. Krunz. This work was supported by the National Science Foundation under Contract ANI-0117840.

The authors are with the Broadband and Wireless Networking Laboratory, School of Electrical and Computer Engineering, Georgia Institute of Technology, Atlanta, GA 30332 USA (e-mail: mcvuran@ece.gatech.edu; ian@ece.gatech.edu).

Digital Object Identifier 10.1109/TNET.2007.893202

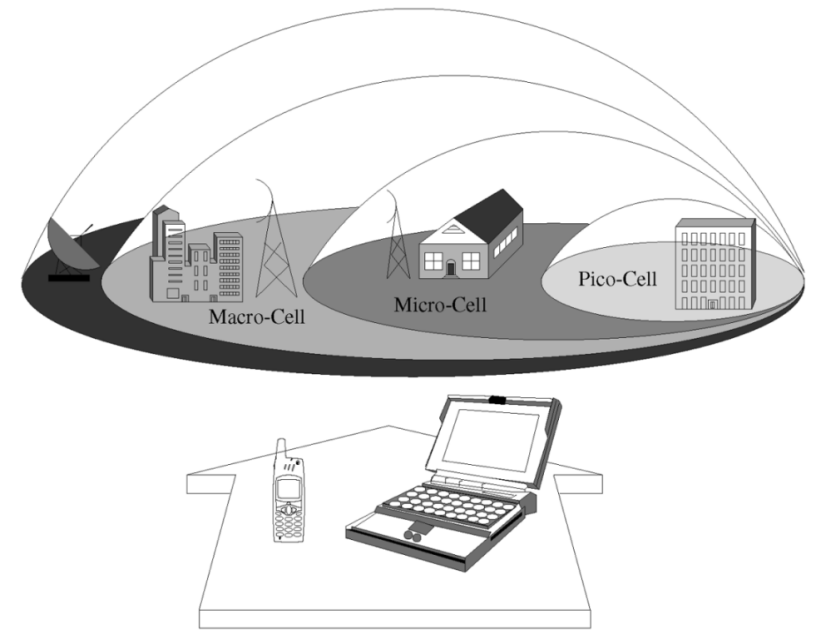

Fig. 1. Next generation wireless networks.

of MAC protocols can be classified in terms of their multiple access schemes. Second-generation systems, e.g., IS-54 and GSM, support TDMA while IS-95, IS-136 and PDC support CDMA [2]. Wideband CDMA (WCDMA) is also designated as the access technology for cdma2000 [30] and UMTS/IMT-2000 [21]. IEEE 802.11 standard for local area networks (LAN) uses CSMA/CA in addition to polling [12]. Different combinations of TDMA, CDMA and WCDMA have also been proposed in the literature [2], [11], [24]-[26]. NG wireless terminals must provide seamless access to these networks, considering the specialized services of different networks.

In addition to the heterogeneity in the medium access schemes of the existing wireless architectures, NG wireless networks are also expected to provide the mobile users with diverse set of services ranging from high rate data traffic to time-constrained real-time multimedia [15]. In [29], four Quality of Service $(\mathrm{QoS})$ classes are defined for UMTS networks, i.e., conversational, streaming, interactive and background. Conversational and streaming classes are mainly intended to be used to carry real-time traffic flows. Conversational real-time services, such as video telephony and voice over IP, are the most delay sensitive applications so the restrictions on transfer delay and jitter are very strict. Unlike conversational class, streaming class such as live broadcasting is a one way transport and has less strict delay requirements than the conversational class. Interactive and background classes are mainly used by traditional internet applications such as web browsing, email, telnet and FTP. Interactive class is characterized by the request response pattern of the end user. In this class, the data is expected to be delivered within a certain time. Examples are interactive web 
browsing, telnet and FTP. On the contrary, the background class does not require the data be transmitted within a certain time. Examples are background delivery of E-mails, short message service (SMS), download of databases and reception of measurement records. In addition to the UMTS network, voice over WLAN (VoWLAN) applications are also gaining interest due to the high popularity of WLANs. However, assuring high quality voice communication through WLANs require QoS-aware networking protocols. Consequently, the scarcity of the wireless resources necessitates efficient utilization of the available bandwidth in NG networks [11]. Therefore, to address the diverse QoS requirements, NG wireless terminals must be able to adapt to the heterogeneous access schemes.

In this paper, we aim to integrate the existing wireless architectures without requiring any modifications in the base stations. Instead, we achieve adaptivity to the architectural heterogeneity as well as diverse QoS requirements by deploying a new adaptive MAC framework in the NG wireless terminals. The challenges addressed in this work can be summarized as follows:

- Heterogeneity in Access Schemes: As explained before, NG wireless terminals encounter different access schemes while roaming between different wireless networks. For a seamless integration, the mobile terminal must be capable of accessing each network when needed.

- Heterogeneity in Resource Allocation: Each network structure performs resource allocation according to various techniques such as TDMA slots, CDMA codes, and random allocation. A metric to compare the amount of the resource allocated by different networks is required to achieve high network utilization in accessing different networks. However, there exists no such unified metric for comparison of the allocated resources by different access schemes.

- Heterogeneity in QoS Requirements: NG wireless terminals are envisioned to provide QoS guarantees according to the underlying network structures. Thus, the MAC layer must efficiently evaluate the available resources in different networks and perform access such a way that the QoS requirements of applications are satisfied.

In order to address these heterogeneities posed by the NG wireless networks, we propose a new two-layer Adaptive Medium Access Control (A-MAC). A-MAC first provides procedures for detecting and accessing the available networks that the NG wireless terminal can access, i.e., access. Then, the available resources in these various types of networks are modeled based on a unified resource model. Each flow that is sent to the MAC layer is then served through the network that is most suitable for the QoS requirements of the flow, i.e., decision. Moreover, A-MAC provides QoS-based scheduling for multiple flows assigned to the same network, i.e., scheduling. As a result, the two-layer A-MAC exploits the available resources in the NG wireless networks by providing procedures for serving multiple flows through multiple network architectures available to the terminal simultaneously.

The main structure and the components of A-MAC are shown in Fig. 2. The access sub-layer is specialized for accessing the network, while the master sub-layer performs decision and scheduling of various application requests for the most efficient

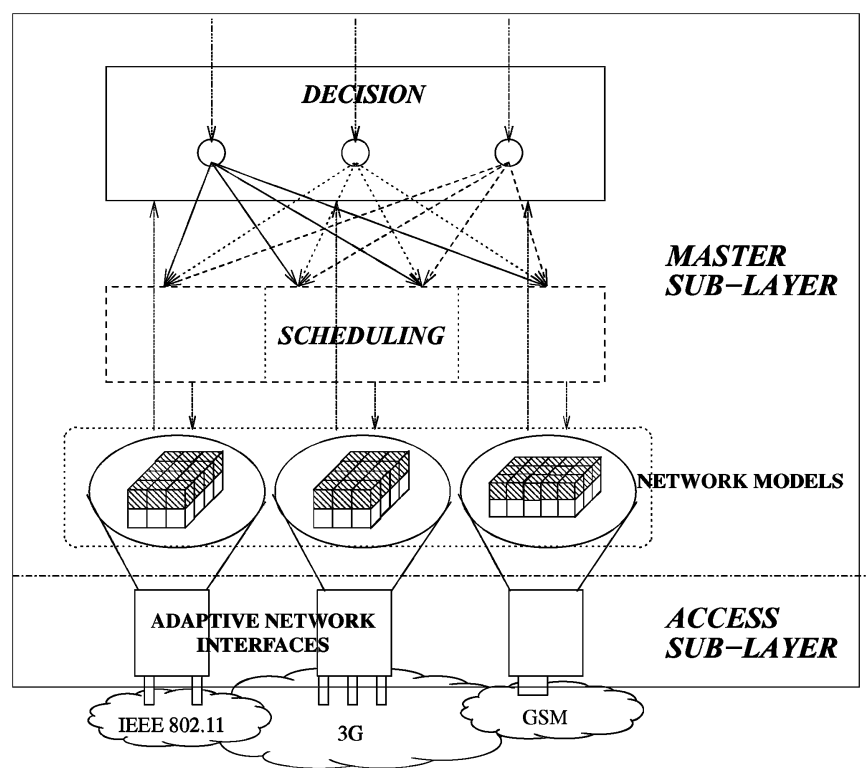

Fig. 2. Main components of A-MAC.

network. A-MAC achieves adaptivity to both the underlying network structure and the QoS requirements of different traffic types. We introduce a novel Virtual Cube concept which serves as a basis for comparison of different network structures. Based on the Virtual Cube concept, A-MAC provides architecture-independent decision and QoS based scheduling algorithms for efficient multi-network access. Incompatibility among medium access and resource allocation techniques are melted into a unified medium access control framework, providing self-contained decision flexibility as well as capability to access various networks.

The rest of the paper is organized as follows. In Section II, the related work on MAC in heterogeneous wireless networks is presented. We introduce the Virtual Cube concept in Section III. Based on the Virtual Cube concept, we explain network modeling in Section IV. The A-MAC is introduced in Section V. We then discuss the performance of A-MAC in Section VI and conclude the paper in Section VII.

\section{RELATED WORK}

There exist several studies in the literature to address the integration of existing wireless systems. In [19], a unified framework for the channel assignment problem in time, frequency, and code domains is proposed. The unified (T/F/C)DMA algorithm consists of labeling and coloring phases. Using the graph theory solutions, channel assignment problems in heterogeneous network structures have been addressed. Although this work provides the fundamental theoretical results for channel assignment in (T/F/C)DMA networks, the framework is not applicable to the existing network structures where the channel assignment principles have already been decided. The application of this framework requires major modifications in the NG wireless network components.

An Ad-hoc CEllular NETwork (ACENET) architecture for $3.5 \mathrm{G}$ and $4 \mathrm{G}$ mobile systems is proposed in [25], where a heterogeneous MAC protocol to integrate IEEE 802.11, Bluetooth 
and HiperLAN/2 with cellular architectures is presented. The coordination between transmissions of different access protocols is provided using beacons from the base stations. ACENET consists of a cellular network and an ad hoc network. Although it is argued that ACENET improves the throughput performance over the existing networks, many modifications in the base stations are required to achieve this.

In [26], a TCDMA protocol is presented for NG Wireless cellular networks. A time-code structure is proposed with subintervals allocated for different functions to enable the integration of cellular networks, ad hoc networks and wireless LANs. However, the integration requires modifications in the existing base stations.

A QoS-oriented access control for the 4G mobile multimedia CDMA communications is presented in [11]. The proposed MAC protocol exploits both time-division and code-division multiplexing. A certain QoS level is guaranteed using fair packet loss sharing (FPLS) scheduling. The proposed MAC protocol is shown to provide QoS guarantees in hybrid TD/CDMA systems. However, the proposed protocol necessitates a new wireless network infrastructure with new base stations for $4 \mathrm{G}$ communications.

In [17], a multiple access protocol is proposed for cellular Internet and satellite-based networks. The proposed scheme accesses to the network with a probability $p_{\text {access }}$ to decrease collision probability based on the network load. However, the QoS requirements of the application are not addressed. Although the proposed scheme is designed for both cellular and satellite networks, it requires modifications in the base station. Hence, the existing cellular networks cannot be used for integration.

As a result, the existing proposals [11], [17], [25], [26] require either a significant modification in the existing infrastructure and base stations or a completely new architecture. Therefore, these approaches lead to integration problems in terms of implementation costs, scalability and backward compatibility. The NG wireless networks are also expected to provide diverse range of services. Such diversity in the services requires adaptivity in the MAC layer in terms of guaranteeing QoS requirements in wireless environments.

In addition to the proposed MAC protocols, there has been extensive research on the physical layer of NG wireless terminals. It is envisioned that $\mathrm{NG}$ wireless terminals will be equipped with multiple-mode radio capabilities. Recent developments in radio receiver and transmitter development have led the way to mobile hand-held devices that are capable of functioning in multiple access technologies. In [1], a dual band (CELL/PCS) triple mode (CDMA/AMPS/TDMA) transmitter has been developed. In [27], an RF module with dual band dual mode (GSM/WCDMA) transmitter and receiver has been developed. In our paper, we assume that NG wireless terminals are capable of receiving signals from multiple network access points and transmitting signals to different access schemes simultaneously.

\section{The Virtual Cube Concept}

NG mobile terminals will encounter different access schemes during accessing different networks within the NG wireless architecture as shown in Fig. 1. Hence, different resource allocation units such as CSMA random access, TDMA time slots,

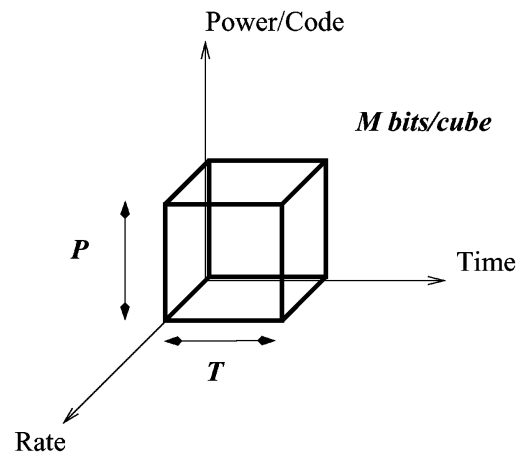

Fig. 3. Virtual cube model.

CDMA codes, as well as hybrid types will be allocated to the mobile terminals (MTs). Thus, MTs must be able to compare these resources in order to provide $\mathrm{QoS}$ guarantees by accessing the most efficient network for the application. However, it is impractical to perform comparison between the resources available to the MT due to the lack of a unified metric for such comparison. For this purpose, we introduce a three-dimensional space model for modeling network resources. Based on this three-dimensional resource-space, we propose a novel Virtual Cube concept in order to evaluate the performance of each network in the reach of an MT. The Virtual Cube concept defines a unit structure based on the resource allocation techniques used in the existing networks. In the following two sections, we describe the resource-space model and the properties of the Virtual Cube, respectively.

\section{A. Resource-Space}

We model the resource in a three-dimensional resource-space with time, rate, and power dimensions as shown in Fig. 3.1 The three dimensions of the resource-space are as follows:

1) Time Dimension: The time dimension models the time required to transfer information.

2) Rate Dimension: The rate dimension models the data rate of the network. Thus, the capacity of different networks with the same connection durations but different data rates are captured in the rate dimension. Furthermore, the bandwidth increase due to the multi-code transmissions or multi-channel communication is also captured in this dimension.

3) Power Dimension: The power dimension models the energy consumed for transmitting information through the network. Note that, the resource in terms of available bandwidth can be modeled using the time and rate dimensions. However, the cost of accessing different networks vary in terms of the power consumed by the wireless terminal. Hence, a third dimension is required. Each network type requires different power levels for transmission of the MAC frames because of various modulation schemes, error coding and channel coding techniques. As a result, the resource differences in these aspects are captured in the power dimension.

${ }^{1}$ Note that the resulting structure may not necessarily be a cube. However, we refer to the concept as virtual cube for ease of illustration. 
The three-dimensional resource space described above enables modeling the available resource from heterogeneous networks as will be explained in Section IV.

\section{B. Virtual Cube Structure}

The virtual cube constitutes the unit structure for modeling and comparing different networks for the appropriate access decision by the NG wireless terminal. The resource space and the virtual cube structure are shown in Fig. 3. The virtual cube structure is defined by three parameters explained as follows:

Cube Capacity (M bits/cube): The number of bits a cube carries.

Cube Duration ( $\mathbf{T}$ sec): The time a cube fills in time dimension. As a result, a virtual cube models a rate of $M / T$ bits/s in the rate dimension.

Cube Power (P Watts): The minimum power for a transmission of a cube.

The three parameters that define the virtual cubes, i.e., $M, T$, and $P$ are fixed for each cube and provide a granularity in each dimension. As will be explained in Section IV, the virtual cubes are used to model the available resource a network provides. In order to model both the available bandwidth and the cost of communication in terms of energy consumption, two types of virtual cubes are used in the A-MAC as described below:

Virtual Information Cube (VIC): VICs model the information sent through the network. They virtually contain $M$ bits and serve as a basis to determine the capacity of the network.

Virtual Power Cube (VPC): The required power consumption for transmission of a bit is different for every network. VICs model the minimum power consumption required to transmit a bit by $P / M$. In order to model the additional power consumption per bit for each network, VPC is used. VPCs model the additional power needed to transmit $M$ bits of information. As a result, VPCs, which virtually contain no data, are used to capture the additional power requirements of a particular network.

\section{NeTWORK MODELING}

The Virtual Cube concept introduced in Section III forms a basis for modeling different access schemes. Based on the Virtual Cube concept, the underlying access schemes are modeled as a three-dimensional structure called resource bin. Each dimension of a resource bin defines the number of virtual cubes that can be filled in that dimension as shown in Fig. 4, where it is shown that VPCs, i.e., gray cubes, are used to model the additional power requirement of the network, while VICs, white cubes, model the available capacity in the network. As a result, resource bins capture the capacity of the network access unit as well as timing information, data rate and power requirements. The modeling of TDMA, CDMA, and CSMA based networks as well as multi-rate communication into resource bins are illustrated in Fig. 4 and explained in the following sections.

\section{A. TDMA Modeling}

In TDMA systems, the channel resource is partitioned into frames. Each frame is further divided into time slots, which are assigned to different users. A TDMA slot is characterized by its slot duration $T_{s}$ and transmission rate $R$. The slot duration, $T_{s}$, specifies the duration of time the MT has the access to the TDMA network within a time frame. The transmission rate, $R$, specifies the rate at which the MT should send packets during the time slot. Due to different modulation schemes, error coding and channel coding techniques, each interface is characterized by an average energy per bit $E_{b}$ to be used during the transmission.

Using these properties, the TDMA slot can be modeled in the resource space. Let $N_{t}, N_{r}$, and $N_{p}$ be the three dimensions of a resource bin representing the number of virtual cubes that can be filled in the time, rate, and power dimensions, respectively. Then, $N_{t}, N_{r}$, and $N_{p}$ can be expressed by

$$
N_{t}=\frac{T_{s}}{T}, \quad N_{r}=\frac{T R}{M}, \quad N_{p}=\frac{E_{b} M}{P}
$$

where $M$ is the cube capacity, $T$ is the cube duration and $P$ is the cube power as described in Section III. A sample model of a TDMA network is illustrated in Fig. 4(a). Note that, if the number of cubes in the power dimension exceeds one, i.e., $N_{p}>$ 1 , VPCs are filled in the power dimension.

\section{B. CDMA Modeling}

In the direct sequence-CDMA (DS-CDMA), each bit of duration $T_{b}$ is coded into a pseudo-noise code of $N$ chips of duration $T_{c}=T_{b} / N$. The pseudo-noise codes used in CDMA are characterized by the processing gain or so-called the spreading gain $G_{p}$ which is expressed as $G_{p}=W / R$ [23], where $W$ is the bandwidth of the CDMA system and $R$ is the data rate. Since each user interferes with other users at the base station in the reverse link, the base station performs power control and assigns a transmission signal power for each MT to utilize the network. The transmitted energy per bit, $E_{b}$, can be expressed as [23], $E_{b}=P_{s} / R$, where $P_{s}$ is the transmitted signal power and $R$ is the data rate.

Although the physical channel is identified by a specific rate of chips, the actual bit rate of the channel varies according to the spreading factor used in coding. The MT is interested in the actual data transmission rate $R$ rather than the channel transmission rate. The data rate of the connection is calculated according to the code assigned to the MT. Moreover, the MT is informed of the power level associated with the transmission of the signal by the base station. In TDD CDMA systems such as UMTS, the time is divided into radio frames, slots and sub-frames. Using the length of the allocated slot, $T_{\mathrm{s}}$ can be determined. In FDD systems, $T_{s}$ is determined by the duration of the connection. Using this information, $N_{t}, N_{r}$ and $N_{p}$ are determined by (1).

In CDMA networks, two nodes may lead to intracell interference when non-orthogonal codes are assigned. However, it is clear that this interference cannot be deterministically calculated before APs assign the codes to nodes and nodes start transmitting information. As a result, in A-MAC, the CDMA network is modeled based on the assignment information provided by the BSs to the adaptive network interfaces (ANIs) as discussed in Section V-C. However, as will be explained in Section V-B, the wireless channel aware scheduling block adjusts the rate of each 


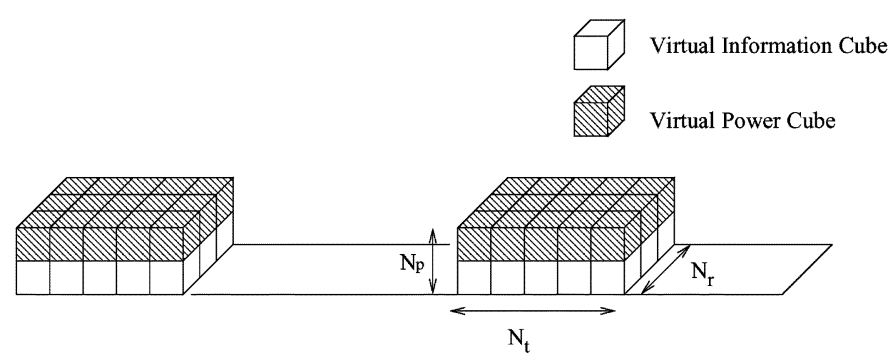

(a)

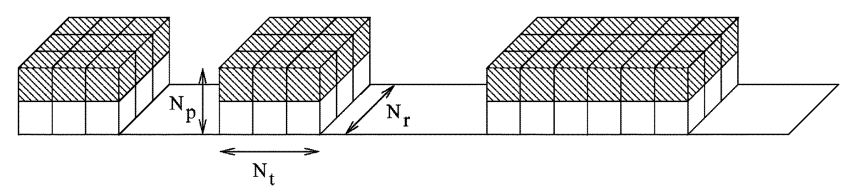

(b)

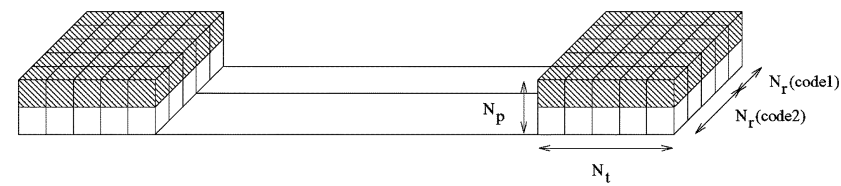

(c)

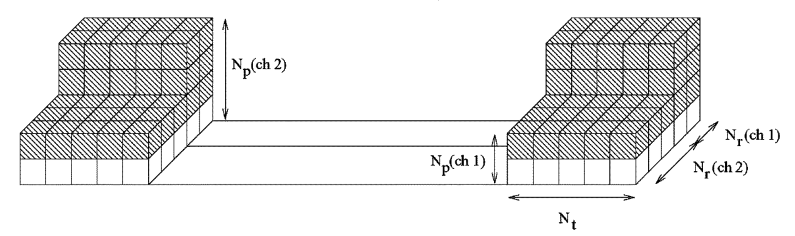

(d)

Fig. 4. (a) TDMA and CDMA modeling. (b) CSMA modeling. (c) Multi-code CDMA modeling. (d) Multi-channel modeling.

flow based on the wireless channel state. As a result, the effects of intracell interference are addressed by the A-MAC protocol.

\section{CSMA Modeling}

CSMA based systems such as IEEE 802.11 are characterized by the randomness in the access to the network. The mobile terminals contend for the channel using CSMA/CA. The MT that captures the medium transmits information. Hence, it is not possible to deterministically calculate the transmission time of a specific MT in CSMA based systems. In a recent work, it has been shown that in wireless networks, the past throughput value has a strong influence on the future throughput value [4]. Moreover, it has been found that irrespective of the velocity of wireless node, throughput prediction based on the past values is feasible. In this respect, various prediction methods can be used for predicting the connection opportunities in CSMA based network. Consequently, for CSMA-based networks, we use the last transmission information to model the resource bin as illustrated in Fig. 4(b). Based on the previous transmission information, e.g., data rate, connection time, consumed power, the dimensions of the model are determined using (1). Moreover, the generated model is dynamically modified to account for wireless medium errors and actual changes in the connection information as explained in Section V-B. As a result, the CSMA-based networks are modeled based on the previous connection information with adaptive updates.

\section{Multi-Rate Networks}

In addition to the modeling schemes for each type of network presented above, a specific network may provide multiple rates to a wireless terminal through various techniques such as additional codes or multi-channel communication. Using the virtual cube concept, multi-rate networks can also be modeled.

In CDMA-based networks, in addition to fixed code assignment, the MT can be assigned multiple codes throughout the communication. Two techniques used in CDMA-based systems for multi-code transmission and modeling of these cases are discussed next.

In IS-95-B and cdma2000, an MT is provided the supplemental code channels (SCC) in addition to a fundamental code channel (FCC) [14], [30]. In IS-95-B, the SCCs have the same spreading gain as the FCC. As a result, multi-code channels provide an aggregate bandwidth for an MT using the same variable spreading gain. In modeling these systems, the rate dimension is extended to include the additional bandwidth provision of multi-code channels as shown in Fig. 4(c).

In addition to code aggregation, variable spreading gain is also used in the cdma2000 system [14], [30]. By reducing the spreading gain, the transmission gain is increased to support high data rate applications. In the cdma2000 system, in addition to an FCC, one SCC is assigned to an MT per data service. Multiple SCCs are used to support multiple data streams with different QoS requirements [14]. The increase in the data rate due to variable spreading gain is also captured in the rate dimension of the resource bin for SCCs as shown in Fig. 4(c).

The number of virtual cubes in the rate dimension, $N_{r}$, of the CDMA frame model increases with the data rate. On the other hand, for a specific bit-error rate (BER), a decrease in the spreading gain increases the average power level [24]. The power level $P_{v}$ for a CDMA signal with a spreading gain $G_{v}$ can be expressed as $P_{v}=P_{f}\left(G_{f} / G_{v}\right)$, where $P_{f}$ is the power level of the fundamental spreading gain and $G_{f}$ is the fundamental spreading gain. Since the rate dimension captures the effect of multi-code transmission in CDMA networks, the number of VPCs in the power dimension, $N_{p}$, remains the same.

Recently, multi-channel communication has gained interest due to the recent advances in transceiver technology. Especially, in cellular networks and IEEE 802.11 standard, multi-channel communication is possible. Note that the number of virtual cubes in the rate dimension, $N_{r}$, depends on many channel and transceiver dependent parameters such as the channel bandwidth, modulation and channel coding schemes. Hence, the resource in each channel needs to be calculated separately as shown in Fig. 4(d). Over a fixed interval, the available aggregate resource through multi-channels is then considered for decision as explained in Section V.

\section{A-MAC: AdAPtive Medium AcCess Control}

A-MAC accomplishes the adaptivity to both architectural heterogeneities and diverse QoS requirements using a two-layer structure as shown in Fig. 2. The access sub-layer is specialized for accessing the network, while master sub-layer performs coordination of the application requests. The access sub-layer con- 
sists of ANIs that are responsible for the adaptivity of the MT to the underlying heterogeneous architectures. The MT communicates with different networks through ANIs. In addition to accessing functionalities, the ANIs provide the environmentawareness to the master sub-layer. Using the information gathered from the underlying network from access points (APs), an ANI models the access scheme and informs the master sublayer. The network modeling, which is explained in Section IV, is performed according to the Virtual Cube concept, which is introduced in Section III.

The master sub-layer consists of scheduler and decision blocks. In NG wireless networks, multiple flows with various quality of service (QoS) requirements can be forwarded to the MAC layer simultaneously. The master sub-layer aims to forward these flows to appropriate interfaces to effectively utilize the wireless medium and guarantee the QoS requirements of each flow. In addition, as the MT traverses through different networks, the most efficient access point for specific QoS requirements is chosen. When a flow is forwarded to the MAC layer by upper layers, the QoS-based decision block first performs decision according to the information about the underlying networks gathered from the ANIs. On top of each interface, a QoS-based scheduler handles fair share of the bandwidth if multiple flows are serviced through the same interface. The functions of decision and scheduler blocks, and the adaptive network interfaces (ANIs) are explained in Sections V-A, V-B, and V-C, respectively.

\section{A. Decision}

NG wireless networks are envisioned to provide diverse set of services which require the MAC layer to efficiently address the QoS requirements of these services. In order to guarantee QoS requirements of each traffic forwarded to the MAC layer, A-MAC performs QoS-based decision for the appropriate interface to forward a specific traffic. This decision is performed by the decision block. The ANIs associated with each network models the available resources based on the principles introduced in Section IV. The available connections are then informed by the associated ANIs to the decision block as shown in Fig. 2. This information consists of the start time of the network slot and the three dimensions of the resource bin, $N_{t}, N_{r}$ and $N_{p}$, which are expressed by (1). The decision algorithm performs decision in each decision interval $T_{d}$ where A-MAC decides which traffic flow will be served on which interface according to the resource bins provided by the ANIs.

A specific network interface $k$ with $k \in C$, where $C$ is the set of active connections, is modeled by the following parameters:

$N_{t}^{k}$ number of information cubes in the time dimension of the network model of interface $k$ during decision interval $T_{d}$;

$N_{r}^{k}$ number of information cubes in the rate dimension of the network model of interface $k$ during decision interval $T_{d}$;

$N_{p}^{k} \quad$ number of power cubes in the power/code dimension of the network model of interface $k$ during decision interval $T_{d}$.
For a specific traffic flow $f$, the decision block chooses the interface such that the utility function, $U_{f}$, given by

$$
U_{f}=\frac{r_{f}^{k} N_{r}^{k} N_{t}^{k}}{N_{p}^{k}}
$$

is maximized. Note that, the utility function, $U_{f}$, aims to find the interface with maximum throughput capability for the minimum transmission power. The utility function (2) is maximized subject to the constraints

$$
\begin{aligned}
\sum_{k \in C_{a}} N_{p}^{k} & \leq N_{p}^{\max } \\
r_{f}^{k} N_{r}^{k} & \geq N_{r, f},
\end{aligned}
$$

where $r_{f}^{k}$ is the bandwidth share of the traffic $f$ in interface $k$, if multiple traffic types are interleaved into one interface, $N_{p}^{\max }$ is the maximum power dissipation allowed for the decision interval T, and $N_{r, f}$ is the minimum required bandwidth in terms of VICs for the traffic type $f$ in order to guarantee its QoS requirements. $C_{a}$ in (3) is the set of active interfaces that are chosen by the decision block. The constraints in (3) and (4) are chosen such that the chosen interface will not exceed the maximum allowed power consumption of the MT and the minimum required bandwidth is met.

Depending on the number of traffic flows to be served and the number of network interfaces available, A-MAC can be in one of four states. The actions taken in each state is summarized in the following sections.

1) Single Type Traffic-Single Interface (STSI): In this case, A-MAC simply forwards the incoming packets from the upper layers to the active interface as long as (3) and (4) are satisfied.

2) Single Type Traffic-Multiple Interfaces (STMI): The decision layer performs the decision algorithm for each of the interface. If the (3) and (4) are met by one or more interfaces, the one that maximizes (2) is chosen. The incoming packets are then fragmented into MAC frames of the specific interface and sent to the ANI. The specified ANI performs access to the network and transmits the frames.

If the QoS requirements of the traffic type cannot be fulfilled using one interface, the decision block attempts to use multiple interfaces for the flow. The interfaces are sorted in a decreasing order of their utility functions. The decision block tries to fulfill the constraints (3) and (4) using multiple interfaces. In this case, the constraint (4) becomes

$$
\sum_{k \in C_{a}^{f}} r_{f}^{k} * N_{r}^{k} \geq N_{r, f}
$$

where $C_{a}^{f}$ is the set of active interfaces chosen for traffic type $f$. If the QoS requirements are guaranteed by multiple interfaces, the decision block forwards incoming packets in a reverse round-robin fashion to the appropriate network. According to the bandwidth share of the traffic in each interface, a weight, $w_{f}^{k}$ is associated with the interface $k$ such that

$$
w_{f}^{k}=\frac{r_{f}^{k}}{\sum_{k \in C_{a}^{f}} r_{f}^{k}} .
$$


The packets are then fragmented into MAC frames according to the properties of the network interface and sent through ANIs.

3) Multiple Type Traffic-Single Interface (MTSI): In this case, A-MAC first checks if the decision constraints hold for each traffic type, assuming they were sent alone through the interface. The traffic types that cannot be satisfied by the current interface are rejected and the upper layers are notified. A-MAC then performs scheduling among the eligible traffic types in order to serve multiple services through the single interface.

According to the QoS requirements of the traffic types, each eligible traffic type $f$ is assigned a bandwidth share $r_{f}^{k}$ such that $\sum_{f} r_{f}^{k}<\left(\frac{N_{r}^{k} * M}{T}\right)$. The decision block then checks if each flow satisfies (3) and (4). If the requirements are guaranteed for each of the traffic type, the scheduler is informed of the bandwidth shares and MAC frame size of the interfaces. The packets from each flows are then fragmented into MAC frames and sent to the scheduler associated with the ANI responsible for the transmission.

If the requirements cannot be fulfilled, the traffic type with the lowest priority is rejected and the bandwidth shares $r_{f}^{k}$ are updated. The algorithm performs the decision algorithm until it finds a set of traffic types that can be served through the interface. Since traffic types that can be served through the interface alone are taken into account, the decision block eventually finds a set of traffic types.

4) Multiple Type Traffic-Multiple Interfaces (MTMI): In this case, A-MAC performs decision according to the priorities of traffic types. The traffic type $f$ with the highest priority is forwarded to appropriate interface or interfaces according to the principles described in Section V-A-2. When the connections $C_{f}^{a}$ for the traffic type $f$ is decided, the capacities of each interface $k \in C_{f}^{a}$ are updated such that the bandwidth occupied by the traffic type $f$ in each interface $k$ is deducted. The same procedure is repeated for other traffic types in decreasing priority among the remaining capacity. This process is performed until either all traffic types are served or all interfaces are occupied. Remaining lower priority traffic types are rejected.

After the decision process, the bandwidth shares of each traffic type in each interface are provided to the appropriate schedulers; and accordingly the scheduling is performed in the ANIs where multiple flows are directed.

One exception in the decision algorithm is the case where WLAN interfaces are evaluated. As explained in Section V-C, CSMA based systems are modeled reactively, i.e., based on the last transmission information, since the access conditions cannot be predicted in advance. However, it might be the case that this previous transmission information may not be present in the node. This occurs when a node enters the coverage area of a WLAN. Since generally, WLAN provides much better performance than the other network structures in terms of data rate, the decision layer gives precedence to the WLAN over other network structures. When a WLAN interface is informed to the master sub-layer, high priority traffic is forwarded to the WLAN. The decision block then checks if the requirements of the traffic type are guaranteed in each decision interval. If the requirements are not guaranteed, the decision algorithm is performed according to the models generated by the ANI as explained in the previous sections.

\section{B. Scheduling}

In NG wireless terminals, multiple flows with various QoS requirements can be forwarded to the MAC layer at the same time. Due to these various QoS requirements, these flows can be served using different types of network architectures that are most suitable for each flow. During the decision process, the decision block may end up selecting a single network structure for multiple services. Since these services cannot be interleaved into a single MAC frame, a QoS-based scheduler is used for each ANI in order to guarantee the QoS requirements of each flow.

Scheduling in wireless networks is a highly explored area because of its importance in the design of base stations [8], [10], [16]. The recent work aims to perform base station centered scheduling to various access requests from multiple MTs. In NG wireless networks, a scheduler is also necessary at the wireless terminal responsible for transmission of multiple flows interleaved into a single interface. However, the requirements of the scheduler differs from the conventional usage of schedulers in base stations. In designing a scheduler for NG wireless terminals, the following requirements are considered.

- QoS Guarantee: The QoS requirements of each flow should be guaranteed throughout the connection.

- Channel Dependent Scheduling: Since wireless channel conditions change throughout the connection, scheduler should be able to adapt to these changes in order to provide fairness to each traffic flow.

- Dynamic Behavior: Since the number of traffic flows assigned to a network AP may vary during the course of scheduling, the scheduler has to be easily configurable to adapt to these dynamic changes.

- Implementation Complexity: Since the number of simultaneous flows to be served in one connection is limited, a scheduler with low implementation complexity is sufficient in NG wireless terminals.

In order to address the requirements of the scheduler in NG wireless terminals, we propose a scheduling algorithm based on the ideas presented in [5]. We extend the Bin Sort Fair Queuing (BSFQ) scheduler in order to accommodate the unique characteristics of the wireless medium. The scheduling algorithm is presented as follows.

In our scheduling algorithm, the output buffer is organized into $N$ scheduling bins. Each scheduling bin is implicitly labeled with a virtual time interval of length $\Delta$. The scheduling bins are ordered according to their virtual time intervals and served in that order. Only the items from the current scheduling bin are transmitted. A virtual system clock $\tau(t)$, which is equal to the starting point of the virtual time interval of the current scheduling bin at time $t$, is maintained in the scheduler. Therefore, the virtual time clock is a step function and $\tau(t)$ is incremented by $\Delta$ whenever all the packets in the scheduling bin are transmitted.

As explained in Section V-A, for each interface $k$, each traffic flow $f$ is assigned a guaranteed rate $r_{f}^{2}$ such that $\sum_{f} r_{f}<$ $\left(\frac{N_{r} M}{T}\right)$, where $N_{r}$ is the number of VICs in the rate dimension of the resource bin of the interface expressed by (1) in

\footnotetext{
${ }^{2}$ Since each scheduler is associated with one ANI, we drop the superscript $k$ introduced in Section V-A in our analysis.
} 
Section IV, $M$ is the cube capacity and $T$ is cube duration as presented in Section III.

It is important to note that, the output link rate may change during the connection time due to wireless channel conditions. This leads to unfairness for the flows which experience channel errors. Thus, our scheduling algorithm adaptively varies the overall output rate according to the available bandwidth. There exist many work on opportunistic wireless link scheduling [3], [6], [9], [20]. The main principle of the wireless link awareness in A-MAC is based on the credit method introduced in [3]. Each flow rate $r_{f}$ is updated if the interface experiences unfairness. The scheduler keeps track of the bandwidth degradation of each flow according to wireless channel errors. This degradation is added to the bandwidth share of the flow. In order to prevent fluctuations in the bandwidth share of flows, the scheduler updates each bandwidth share in each decision interval.

Each packet forwarded to the scheduler is fragmented into MAC frames according to the MAC frame structure of the network. The $j$ th frame of flow $f$, denoted by $p_{f}^{j}$, is assigned with the virtual time stamp $v t s\left(p_{f}^{j}\right)$ such that

$$
v t s\left(p_{f}^{j}\right)=\max \left(\tau\left(A\left(p_{f}^{j}\right)\right), v t s\left(p_{f}^{j-1}\right)\right)+\frac{l_{f}}{r_{f}}, \quad j \geq 0
$$

where $\tau\left(A\left(p_{f}^{j}\right)\right)$ is the system virtual time at the arrival time of frame $p_{f}^{j}$, and $l_{f}$ is the length of the frame $p_{f}^{j}$ which is specified by the resource bin, i.e., $l_{f}=M N_{t} N_{r}$. Arriving frames are then stored in their corresponding scheduling bins in the FIFO order. The index $i_{f}^{j}$ of the scheduling bin to store frame $p_{f}^{j}$ is equal to

$$
i_{f}^{j}=\left\lfloor\frac{v t s\left(p_{f}^{j}\right)-\tau\left(A\left(p_{f}^{j}\right)\right)}{\Delta}\right\rfloor, \quad j>0 .
$$

If $i_{f}^{j}=0$, then $p_{f}^{j}$ is stored in the current scheduling bin. If $i_{f}^{j}<N$, it is stored in the $i_{f}^{j}$ th scheduling bin following the current scheduling bin. If $i_{f}^{j} \geq \mathrm{N}$, the frame is discarded. As a result, packets from different flows are scheduled in a frame-byframe basis.

The original BSFQ algorithm provides fairness guarantee based on the values of maximum packet length $l_{f}^{\max }$ and guaranteed data rate $r_{f}$ (Please refer to Theorem 2 in [5]). Note that, in A-MAC, the BSFQ algorithm is enhanced to integrate the procedures for determination the packet size and data rate with our virtual cube concept. Other than this modification, the functionality of the BSFQ algorithm is preserved. Since the fairness guarantee is a function of the $l_{f}^{\max }$ and $r_{f}$, the fairness guarantee provided by [5] is still valid in our scheduler.

\section{Adaptive Network Interfaces}

When a flow is forwarded to the A-MAC protocol, the decision block performs QoS-based decision to select the appropriate interface for the data transfer as explained in Section V-A. In the case of multiple flows assigned to a single interface, the QoS-based scheduler explained in Section V-B in each interface orders the packets accordingly. Finally, the MAC frames are forwarded to the Adaptive Network Interfaces (ANIs) in the access sub-layer as shown in Fig. 2. Accessing the networks is accomplished by the ANIs. With the aid of the underlying physical capabilities of the MT, each interface is capable of performing the following functions:

1) Network Structure Awareness: ANIs provide the MT with environment-awareness by gathering information about the underlying network structures. When the MT enters the coverage area of an access point (AP) of a network, the associated ANI gathers network information from the beacons periodically sent from the AP. The environment awareness is, hence, achieved in different wireless systems, i.e., GSM, UMTS, cdma2000, and WLAN, as follows:

GSM: The base stations use Broadcast Control Channel $(\mathrm{BCCH})$ to periodically broadcast information about the properties of a cell for MTs. BCCH is mapped to a specific radio frequency for each cell and it does not hop between radio frequencies. MTs access the network using this channel by decoding the base station identification code and frequency [18], [29]. UMTS: The Broadcast Channel (BCH) is used to broadcast system- and cell-specific information. The $\mathrm{BCH}$ is always transmitted over the entire cell and has a single transport format [28]. cdma2000: The paging channel is used to send common channel information that is used for accessing the network [30]. WLAN: The MT is assumed to listen to the channel in passive mode for a beacon from a WLAN AP. If such a beacon is received, the master sub-layer is informed. When a beacon is received from one of the networks by the specified ANI, the master sub-layer is informed about the existence of an access network.

2) Network Modeling: Once the existence of a network has been informed to the master sub-layer, ANIs model the network MAC structure as explained in Section IV and inform the master sub-layer as shown in Fig. 2. When a mobile terminal enters the coverage area of an AP, the network specific information, such as slot information, data rates and multiplexing information is captured by the ANI. The ANI then, models the access structure according to a minimum resource allocation that is achievable from the network. As the MT is connected to the network, the BS may allocate more bandwidth to the MT according to the QoS requirements of the traffic. In this case, the associated ANI updates the corresponding resource bin according to the allocated resource. Furthermore, as the modeled properties, i.e., data rate, spreading gain or frame dimensions vary during the connection, the ANI updates the resource bin and informs the master sub-layer.

3) Access and Communication: An ANI associated with a network structure performs access to the network if it is selected for a transmission by the master sub-layer. The communication with the AP is performed according to the network specific procedures. Each network has specific procedures for access phase and resource allocation. The packet structures, message formats and signaling procedures already exist for each network. Due to implementation and scalability concerns, it is not feasible to propose modifications to the existing MAC protocols that are deployed in the access points of these networks. Since there exists no unified standard for each network, we assume that the ANIs are embedded with the functionalities of the MAC protocols already deployed for each network required for access 
TABLE I

TRAFFIC MODEL PARAMETERS

\begin{tabular}{|c|l|l|}
\hline \multicolumn{2}{|c|}{ Parameter } & Value \\
\hline & Avg. Talk Duration & $180 \mathrm{~s}$ \\
Voice & Avg. Principal Talkspurt Duration & $1.0 \mathrm{~s}$ \\
Traffic & Avg. Principal Gap Duration & $1.35 \mathrm{~s}$ \\
& Avg. Minispurt Duration & $0.275 \mathrm{~s}$ \\
& Avg. Minigap Duration & $0.05 \mathrm{~s}$ \\
& Packet Length & $260 \mathrm{bits}$ \\
\hline CBR & Avg. Talk Duration & $360 \mathrm{~s}$ \\
Video & Bit Rate & $64 \mathrm{kbps}$ \\
Traffic & Packet Length & $1280 \mathrm{bits}$ \\
\hline & Avg. Talk Duration & $180 \mathrm{~s}$ \\
VBR & Avg. State Holding Duration & $160 \mathrm{~ms}$ \\
Video & Minimum Bit Rate & $120 \mathrm{Kbps}$ \\
Traffic & Maximum Bit Rate & $420 \mathrm{Kbps}$ \\
& Avg. Bit Rate & $239 \mathrm{Kbps}$ \\
& Packet Length & $840 \mathrm{bits}$ \\
\hline \multirow{3}{*}{ Best-Effort } & Avg. Packet Generation Rate & $20 \mathrm{Msgs} / \mathrm{s}$ \\
(ABR) & Avg. Idle Time & $2 \mathrm{~s}$ \\
Traffic & Avg. Message Length & $30 \mathrm{kB}$ \\
& Avg. Frame Length & $150 \mathrm{bits}$ \\
\hline
\end{tabular}

and communication with the APs. Once the underlying network is chosen to access, the corresponding ANI performs the required access and resource allocation procedures for accessing that specific network and transmission.

\section{Performance Evaluation}

In this section, we investigate the performance of A-MAC for different scenarios in CDMA, TDMA and CSMA based networks. In these scenarios, the throughput performance of the proposed framework is analyzed. We investigate the performance of an adaptive NG terminal equipped with A-MAC, in the presence of heterogeneous network architecture. In the simulations, we simulate background traffic using designated nodes for each traffic model and network type. For the A-MAC protocol, decision interval, $T_{d}$, is chosen as $1 \mathrm{~s}$. The traffic models used in the simulations, the network structures, and the results of the performance evaluations are explained in Sections VI-A, VI-B, and VI-C, respectively.

\section{A. Traffic Models}

Four types of traffic models are used in the simulations. We describe each traffic model in the following sections. The values of the traffic model parameters are shown in Table I. Moreover, in Table I, the requirements for each traffic type in each network in terms of BER and timeout are shown.

1) Voice Traffic: Voice traffic is modeled based on a threestep Markov model [2]. The voice traffic is assumed to have main talkspurts and gaps denoted as principal talkspurts and principal gaps, respectively. The principal talkspurt also consists of minispurts and minigaps. The duration of each spurt and gap is exponentially distributed and statistically independent of each other. The talk duration is also exponentially distributed. The average duration of each distribution is shown in Table I. The stations generate IP packets with length of 260 bits in every 20 seconds achieving a constant rate of $13 \mathrm{~kb} / \mathrm{s}$ during the talkspurts.

2) CBR Video Traffic: CBR video traffic is modeled with a constant rate of packets generated with talk duration exponentially distributed.
TABLE II

NETWORK MODEl PARAMETERS

\begin{tabular}{|c|c|c|c|c|}
\hline \multicolumn{5}{|c|}{ TDMA System } \\
\hline BS radius & \multicolumn{4}{|c|}{$25 \mathrm{~m}$} \\
\hline Frame Length & \multicolumn{4}{|l|}{$4.6 \mathrm{~ms}$} \\
\hline Slot Length & \multicolumn{4}{|l|}{$577 \mu \mathrm{s}$} \\
\hline Slot rate & \multicolumn{4}{|c|}{$115.2 \mathrm{kbps}$} \\
\hline Operating Frequency & \multicolumn{4}{|c|}{$900 \mathrm{~Hz}$} \\
\hline & Voice & CBR & \multicolumn{2}{|l|}{ ABR } \\
\hline Max. Slots & 8 & 8 & \multicolumn{2}{|l|}{8} \\
\hline Max. Slots for a MT & 1 & 2 & \multicolumn{2}{|l|}{1} \\
\hline Timeout & $10 \mathrm{~ms}$ & $50 \mathrm{~ms}$ & \multicolumn{2}{|l|}{$1 \mathrm{~s}$} \\
\hline \multicolumn{5}{|c|}{ CDMA System } \\
\hline BS radius & \multicolumn{4}{|c|}{$40 \mathrm{~m}$} \\
\hline Operating Frequency & \multicolumn{4}{|c|}{$1.8 \mathrm{GHz}$} \\
\hline & Voice & CBR & VBR & ABR \\
\hline BER & $10^{-3}$ & $10^{-4}$ & $10^{-6}$ & $10^{-9}$ \\
\hline Max. Codes & 16 & 8 & 4 & 4 \\
\hline Max. Codes for a MT & 1 & 1 & 2 & 1 \\
\hline Timeout & $20 \mathrm{~ms}$ & $50 \mathrm{~ms}$ & $40 \mathrm{~ms}$ & $1 \mathrm{~s}$ \\
\hline \multicolumn{5}{|c|}{ WLAN System } \\
\hline AP radius & \multicolumn{4}{|l|}{$15 \mathrm{~m}$} \\
\hline Transmission rate & \multicolumn{4}{|l|}{$1 \mathrm{Mbps}$} \\
\hline Operating Frequency & \multicolumn{4}{|l|}{$2.4 \mathrm{GHz}$} \\
\hline
\end{tabular}

3) VBR Video Traffic: VBR Video traffic models the videophone and videoconference transmissions with a multistate model [2]. The multi-state model generates continuous packets for a certain duration in each state. The bit rate in each state is determined independently using a truncated exponential distribution. Holding duration of each state is exponentially distributed and statistically independent of each other. The duration of the traffic is also determined according to an exponential distribution.

4) Best-Effort (ABR) Traffic: ABR traffic models the nonreal-time best-effort data traffic. During the ABR connection, multiple packets are sent. The length of each packet is geometrically distributed and independent of each other. The total length of the packets in a connection and the idle time between consecutive connections are exponentially distributed.

\section{B. Network Models}

Three different network structures are simulated throughout the performance evaluation. We describe these network structures in the following sections.

1) TDMA System: A TDMA based system is simulated with time partitioned into time frames. Each time frame is further partitioned into time slots. The time frame length is $4.6 \mathrm{~s}$ with each frame containing 8 time slots. The time slot size is $577 \mu \mathrm{s}$ with a capacity of 156 bits. We simulate a TDMA based system based on GPRS, such that multiple slots are assigned to the MT during one time frame to provide $\mathrm{QoS}$ of different traffic types. Each MT is assigned multiple slots in each frame according to its traffic requirements. The system parameters used in the simulations are shown in Table II. The Maximum Time Slots field denotes the maximum number of slots that can be allocated to a specific traffic type by the BS, where Maximum Time Slots for a MT denotes the maximum time slots that can be allocated to a single MT in a frame.

2) CDMA System: An MC-CDMA system is simulated with time partitioned into uplink and downlink frames. Each uplink 


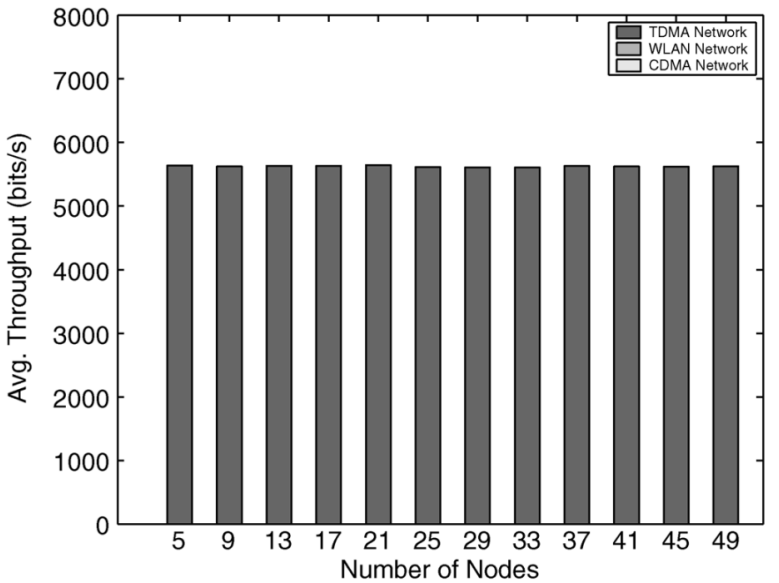

(a)

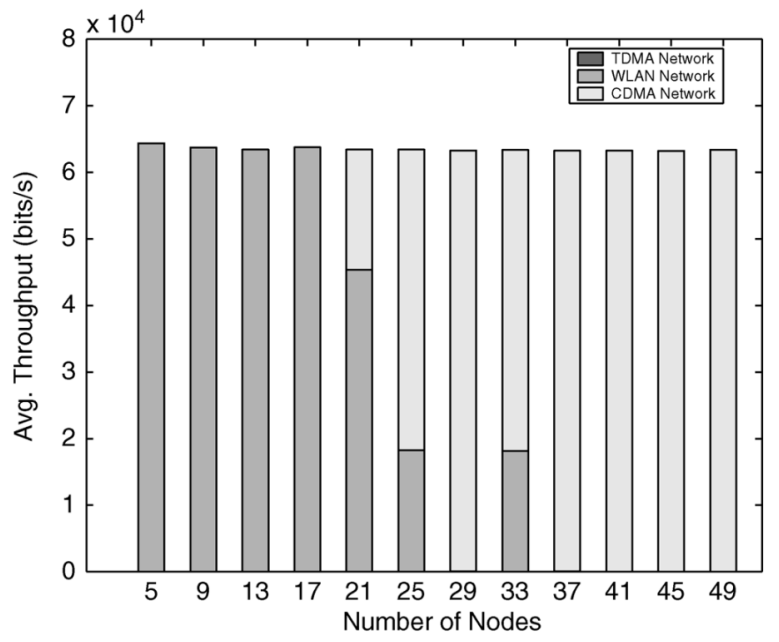

(c)

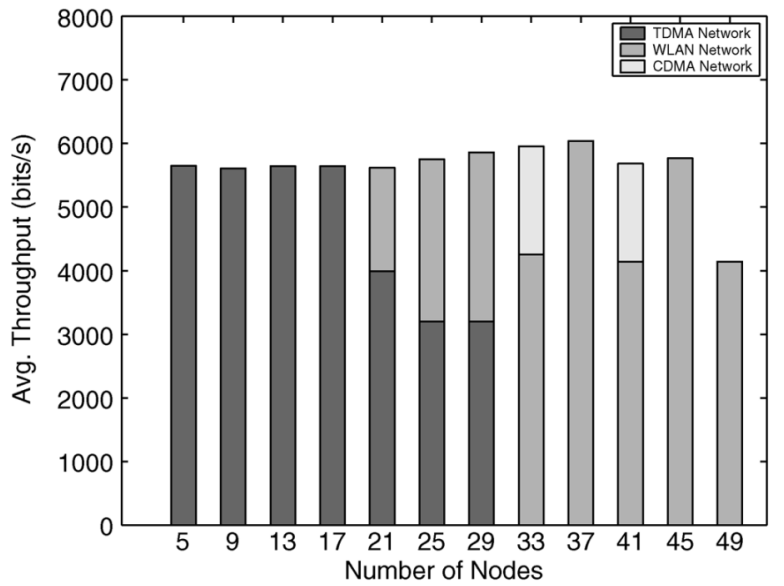

(b)

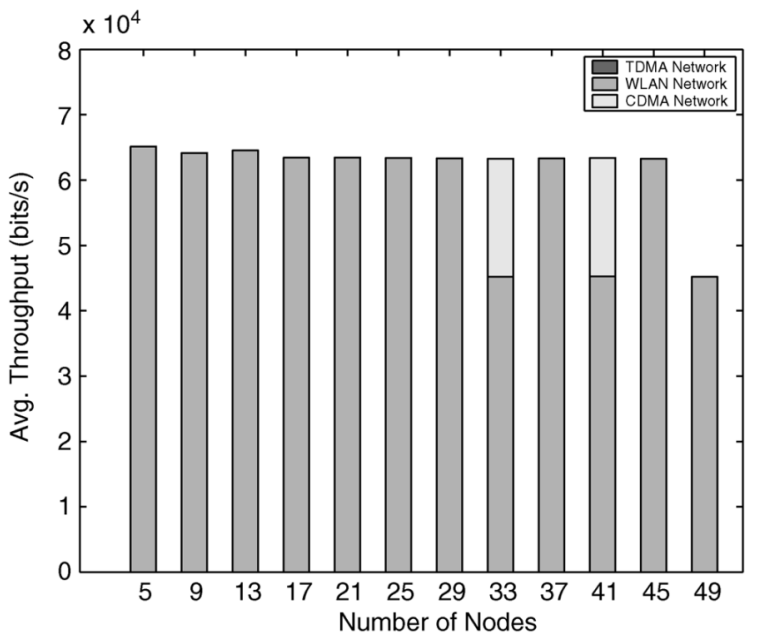

(d)

Fig. 5. Average throughput of voice traffic with $\left(n_{T}, n_{C}, n_{W}\right)=\left(\right.$ a) $(10 \%, 10 \%, 80 \%)$, (b) $(50 \%, 30 \%, 20 \%)$, and CBR traffic with $\left(n_{T}, n_{C}, n_{W}\right)=($ c) $(10 \%$, $10 \%, 80 \%)$, (d) $(50 \%, 30 \%, 20 \%)$.

and downlink frame is $10 \mathrm{~ms}$. A frame is further partitioned into 15 slots. Each slot contains 2560 chips. The CDMA network has a transmit rate of $3.84 \mathrm{MChips} / \mathrm{s}$. Based on the BER requirements and target SINR of each traffic type, multiple codes are assigned to an MT. The network provides the MT up to $960 \mathrm{~kb} / \mathrm{s}$ bandwidth. The base station is assumed to perform power control in each connection and the CDMA system is assumed to perform successful handoff as the mobile terminal roams through the network. The system parameters used in the simulations are shown in Table II.

3) WLAN System: The WLAN system is simulated based on the IEEE 802.11 MAC layer [12]. The MT communicates with the access point of the WLAN network throughout the communication. The transmission rate in the WLAN system is assumed to be $1 \mathrm{Mb} / \mathrm{s}$.

\section{Simulation Results}

The A-MAC protocol is simulated using the traffic models and network systems presented in Section VI-A and Section VI-B, respectively. In a $200 \mathrm{~m} \times 200 \mathrm{~m}$ grid, nodes are placed with uniform distribution. Each node is assumed to be able to connect to only one network. In addition, a node equipped with A-MAC is simulated in the grid. We refer to this node as the Adaptive Node throughout our discussions. We vary the node number in each network and the percentage of traffic distribution to analyze the performance of A-MAC in the heterogeneous network structure. Each simulation lasts $230 \mathrm{~s}$ and the results are average of 5 trials for each 5 random topologies.

Note that, although a single A-MAC node is investigated throughout the simulations, since A-MAC provides seamless access and communication to each of the networks, each connection in the background traffic can also be considered to be generated by another A-MAC node. A-MAC aims to provide a seamless MAC protocol for accessing to heterogeneous networks without requiring any modifications to the existing medium access schemes. As a result, the resource assignment for each connection attempt is handled by the specific principles of the network. Hence, for a single A-MAC node, the effects of other A-MAC nodes are not distinguishable from any node inside a network.

As explained in Section I, in NG networks, adaptive nodes encounter heterogeneity in both network structure and traffic types. In order to investigate the effects of these heterogeneities 


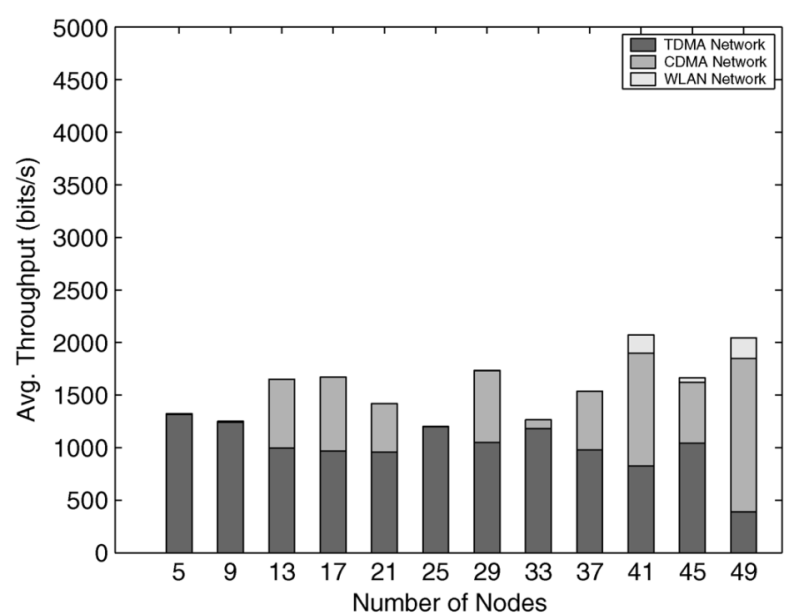

(a)

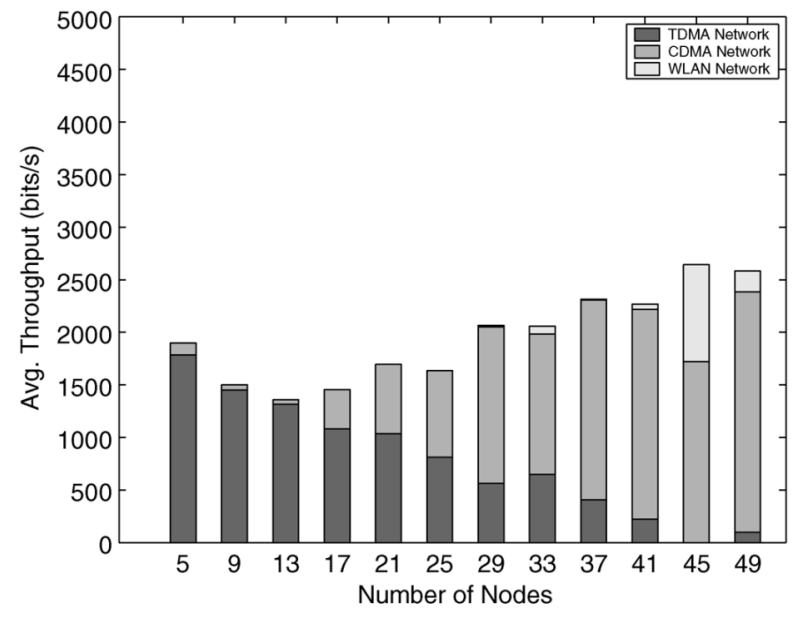

(b)

Fig. 6. Average throughput of best-effort traffic with $\left(n_{T}, n_{C}, n_{W}\right)=($ a) $(10 \%, 10 \%, 80 \%)$, (b) $(50 \%, 30 \%, 20 \%)$.

on the performance of the A-MAC and evaluate the adaptivity of the protocol, we performed two sets of simulations as explained in the following sections.

1) Fixed Topology: In this set of experiments, we are interested in the adaptivity of the A-MAC protocol to the heterogeneity in the traffic load in different networks. Hence, we simulate a fixed topology where all the nodes, including the adaptive node, are stationary. The adaptive node is assumed to be in the coverage area of three of the network structures, i.e., TDMA, CDMA and WLAN access points, ${ }^{3}$ throughout the simulation. Each node in the network creates a specific type of traffic and tries to send it to its designated access point. The traffic type distribution (Voice, ABR, CBR, VBR) is chosen as $(65 \%, 15 \%$, $10 \%, 10 \%)$. The number of nodes in each network is defined by their percentage of the total number of nodes in the simulations. The distribution of the number of nodes in each network is defined as triple $\left(n_{T}, n_{C}, n_{W}\right)$, where $n_{T}, n_{C}$, and $n_{W}$ denote the percentage of nodes in TDMA, CDMA and WLAN networks, respectively. The adaptive node creates four types of the traffic according to the models described in Section VI-A and tries to

\footnotetext{
${ }^{3}$ We use the term access point for both the base stations and the access points throughout the section
}

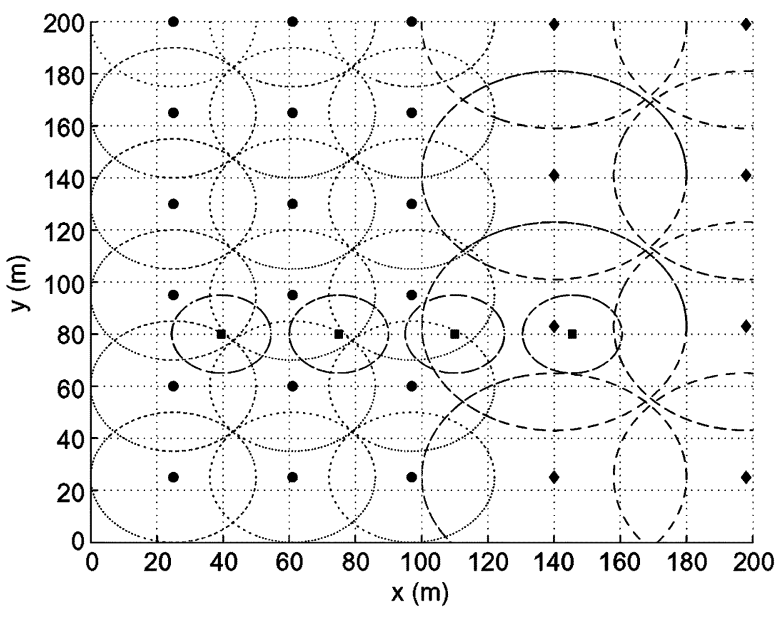

Fig. 7. Sample topology used in the mobility simulations. $\bullet$ : TDMA BS; CDMA BS; $\square$ : WLAN AP.

send it to one of the networks. We performed various simulations with varying load on the networks. The average throughput performance of A-MAC is shown in each case.

In Fig. 5(a) and (b), the average throughput of the A-MAC for voice traffic is shown with two node distributions, i.e., $\left(n_{T}, n_{C}, n_{W}\right)=(10 \%, 10 \%, 80 \%)$ and $(50 \%, 30 \%, 20 \%)$. The horizontal axis represents the total number of nodes creating traffic throughout the simulations in the coverage area of the three network access points. As shown in Fig. 5(a), voice traffic is served through the TDMA network when the traffic load is low in the TDMA network. This decision is due to the capability of TDMA structure to support voice traffic. However, when the load on the TDMA network is increased, as shown in Fig. 5(b), A-MAC uses multiple networks in order to provide the required throughput of the voice traffic. When the number of nodes inside the TDMA network exceeds 10 , (i.e. $21 * 50 \%$ ), $30 \%$ of the voice traffic is served through the WLAN access point. As the load is increased, TDMA network cannot provide the required capacity and A-MAC switches to WLAN network. Fig. 5(b) shows that as the traffic load in WLAN network is increased, CDMA network is chosen in order to achieve the throughput guarantee of the voice traffic. As shown in Fig. 5(b), as long as there is available capacity in the reach of the adaptive node, A-MAC exploits this capacity to serve the required traffic.

In Fig. 5(c) and (d), the average throughput of CBR traffic is shown. In both figures, the required throughput is met for increasing traffic load. In Fig. 5(c), A-MAC accesses WLAN network to serve the CBR traffic when the traffic load is low. When the WLAN traffic load is increased, the CBR traffic requirements cannot be guaranteed, and the CDMA network is chosen. The same behavior is also observed for the VBR traffic, which is not shown here. A-MAC exploits both networks in order to provide the throughput required by the CBR and VBR traffic, i.e., $64 \mathrm{~kb} / \mathrm{s}$ and $239 \mathrm{~kb} / \mathrm{s}$. In Fig. 6(a) and (b), the throughput of the best effort traffic for both of the load distributions is shown. Since the best effort traffic has no bandwidth requirements, it is served as the resources in a network are available. As shown in Fig. 6(b), A-MAC mainly serves best-effort traffic through the TDMA network. However, as the traffic load is increased, CDMA and WLAN networks are used as they have 


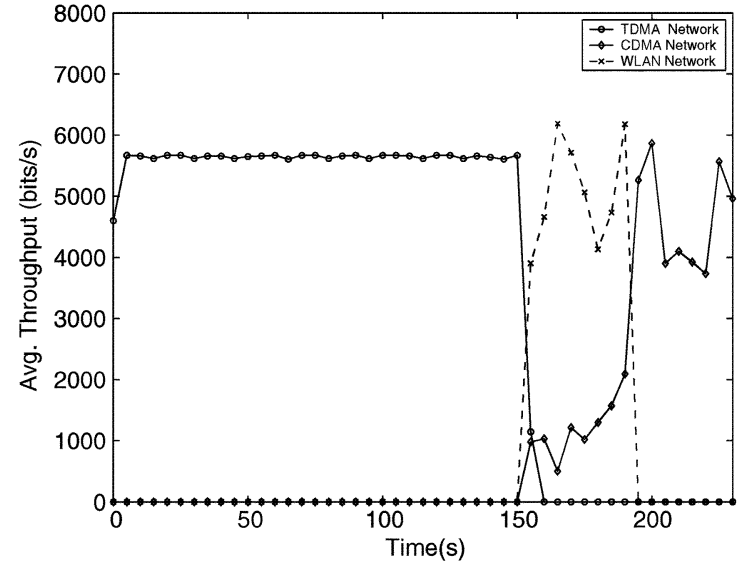

(a)

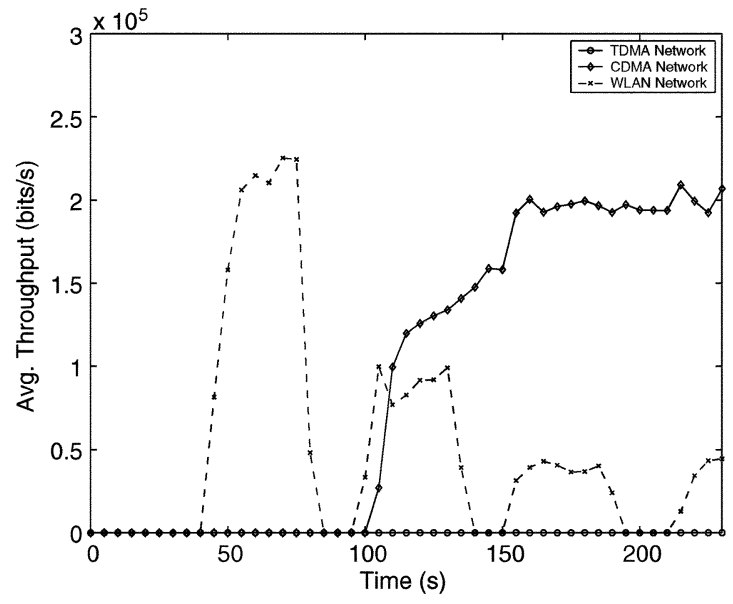

(c)

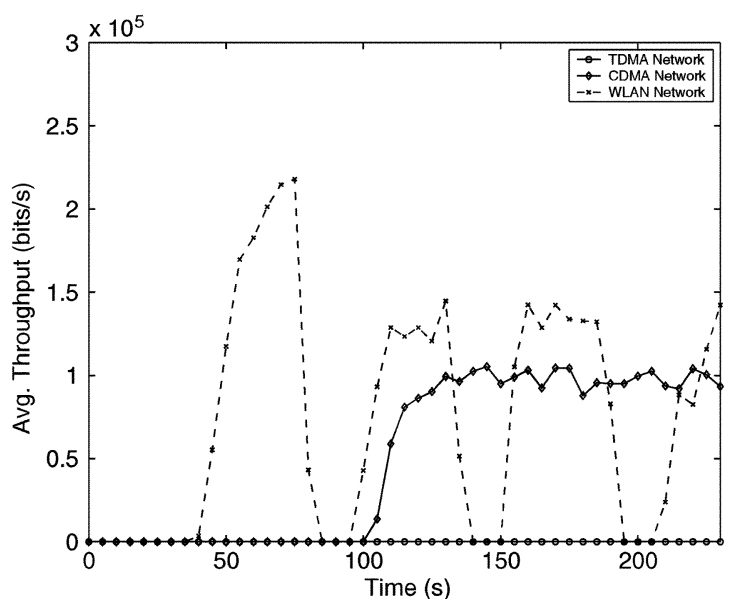

(b)

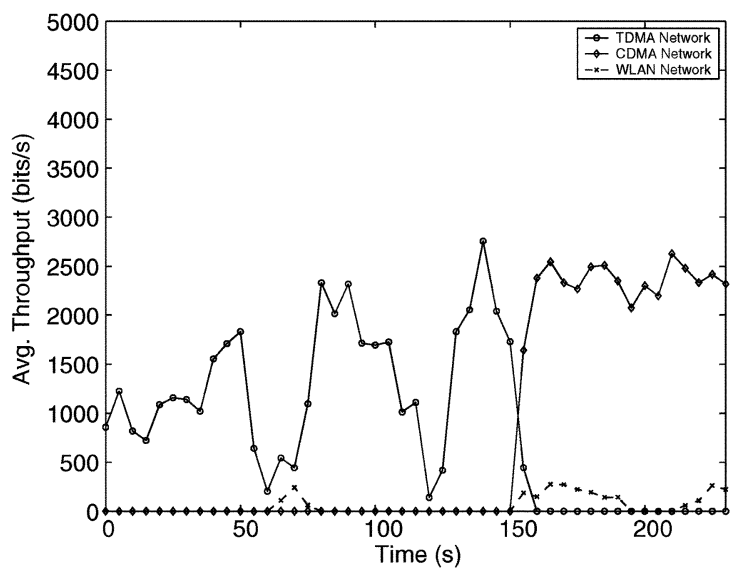

(d)

Fig. 8. Throughput of (a) voice, (b) CBR, (c) VBR, and (d) ABR traffic in dynamic topology.

available bandwidth. The decision is made mainly based on the decision requirements of the mobile station instead of traffic requirements as described in Section V-A. Note that the achieved throughput is actually lower than the average transmission rate of these traffic types. This is due to the channel errors and access collisions, which also increase with increasing traffic load. However, A-MAC exploits the available bandwidth in the remaining networks to guarantee the requirements of the traffic types.

2) Dynamic Topology: In order to investigate the adaptivity of the A-MAC protocol to the heterogeneity in the network environment, we performed a second set of experiments with a dynamic topology. The network topology used in the simulations is shown in Fig. 7. The various points in Fig. 7 represent the TDMA, CDMA and WLAN access points with the circles representing their coverage areas. The topology is designed such that the left of the grid is covered by a TDMA network and the right part is in the coverage area of a CDMA network. WLAN access points are spread over the topology including the overlapping area of TDMA and CDMA networks. This type of topology can simply be thought of as the hot spots in a metropolitan network. In our simulations, we used 100 mobile nodes and a simple mobility pattern for the adaptive node in order to observe the response of the A-MAC to varying network structures as shown in
Fig. 7. The adaptive node roams through TDMA to the CDMA network, passing through the coverage area of WLAN APs. The simulation lasts $230 \mathrm{~s}$. The instantaneous throughput, calculated by averaging the throughput for intervals of $5 \mathrm{~s}$, is shown for each of the traffic types in Fig. 8.

The adaptive terminal serves the voice traffic throughout the simulation, through TDMA, WLAN and CDMA networks as shown in Fig. 8(a). As the adaptive node enters the overlapping area of the networks, the voice traffic is handed over to the WLAN network, since the adaptive node leaves the coverage area of the TDMA network. In addition, part of the traffic is served through the CDMA network in order to achieve the traffic requirements. However, since the TDMA network cannot allocate enough bandwidth to CBR and VBR traffic due to high load in the network, the CBR and VBR traffic are served only through WLAN APs while the adaptive node is in the coverage area of the TDMA network as shown in Fig. 8(b) and (c). As the adaptive mobile node enters the coverage area of the CDMA network, the CBR and VBR traffics are handed over to the CDMA network. In Fig. 8(c), the average throughput of the best-effort traffic is shown. A-MAC serves the best effort traffic through three types of networks throughout the simulations. However, note that the time the adaptive node resides in the WLAN network is also evident from the significant decrease in the best 
effort traffic throughput. As shown in Fig. 8(b) and (c), CBR and VBR traffic are only served through the WLAN APs while the adaptive node is in the coverage area of the TDMA network. Hence, in order to serve these traffic types and meet the decision requirements at the same time, A-MAC suppresses lowest priority best-effort traffic. The decrease in throughput in Fig. 8(c) is due to the priority mechanism of A-MAC. Fig. 8(b) and (c) show that A-MAC provides smooth transition between three network structures as the adaptive node roams through them. The voice traffic requirements are satisfied throughout the simulation, where CBR and VBR traffic are served through the WLAN APs and the CDMA network in the second part of the network, where required bandwidth is provided. As shown in Fig. 8, A-MAC exploits the available bandwidth in its vicinity by performing necessary switches from networks to networks and aggregating available bandwidth via its multiple access capabilities.

In addition to the adaptivity to heterogeneities of the next generation wireless networks, A-MAC also provides a memory efficient access capability to the next generation wireless terminals. It is evident from the discussion in Section I that next generation wireless terminals will be equipped with physical capabilities to access different types of multiple access technologies [1], [27]. Although the physical layer for heterogeneous wireless access is being developed, efficient network protocols are still required for next generation wireless terminals. The main concern in developing protocols for the NG wireless terminals is the memory constraints posed by the mobile devices due to limited memory and processing capabilities. Hence, the research is focused on developing software tools for implementation of protocols with minimum memory footprint [22], in addition to consistent, platform independent software interface layer for mobile application processors, i.e., managed runtime environments (MRTEs) [7], [13]. It is clear that implementing separate protocol stacks for accessing each type of network is contradictory to the memory efficiency of the MAC layer. Such an approach would require excessive memory requirements, independent access protocols and inefficient resource management since these protocols are not developed to coexist in the same device. However, it is also clear that limited access functionalities such as, synchronization procedures, packet structures, are required for access to each network scheme. As a result, A-MAC avoids whole protocol stacks to be implemented to the NG wireless terminals by implementing the required functionalities in the ANIs and performs adaptive access decision by simple decision and scheduling algorithms which provide seamless medium access to the upper network layers.

\section{CONCLUSION}

In this paper, we propose an adaptive medium access control (A-MAC) for NG wireless terminals. To the best of our knowledge, this is the first effort on designing a MAC protocol that achieves adaptation to multiple network structures with QoS aware procedures without requiring any modifications to the existing network structures. A-MAC provides seamless access to multiple networks using a two-layered architecture to achieve adaptation to heterogeneous access networks and QoS requirements from traffic types. The access sub-layer consists of ANIs, which perform network access. The master sub-layer coordinates incoming traffic packets and provides QoS based service using the available access networks in the reach of the MT.

We introduced a novel virtual cube model as a unit structure to compare different access schemes. According to the virtual cube model, access networks are modeled into three-dimensional resource bins by the ANIs. Using these resource bins, the master sub-layer performs QoS-based decision in order to choose the best access network for a specific traffic type. The schedulers associated with each ANI are invoked in case of multiple traffic flows assigned to a single interface. The scheduler performs QoS-based scheduling achieving fairness and delay guarantees on traffic flows according to their priorities. The simulation results confirm that A-MAC satisfies QoS requirements of different traffic types by adapting to the heterogeneity in the network structure and the available resources in each network in its reach.

Note that in our framework we do not consider the cost of switching between network technologies. However, this fact can easily be incorporated into our decision framework. More specifically, if the cost of switching from a specific network is high, the decision interval can be increased such that frequent handovers between networks are prevented. Another approach could be to incorporate a cost function into the network modeling framework provided in Section VI-B. More specifically, a cost function can be incorporated into the network modeling framework as a fourth dimension in the virtual cube concept such that the decision is performed accordingly. These possible solutions definitely introduce a tradeoff between performance and cost, which is a further research topic in our approach.

\section{REFERENCES}

[1] S. Aggarwal et al., "A highly integrated dual-band triple-mode transmit IC for CDMA2000 applications," in Proc. 2002 Bipolar/BiCMOS Circuits and Technology Meeting, 2002, pp. 57-60.

[2] I. F. Akyildiz, D. A. Levine, and I. Joe, "A slotted CDMA protocol with BER scheduling for wireless multimedia networks," IEEE/ACM Trans. Netw., vol. 7, no. 2, pp. 146-158, Apr. 1999.

[3] P. Bhagwat, P. Bhattacharya, A. Krishna, and S. K. Tripathi, "Enhancing throughput over wireless LANs using channel state dependent packet scheduling," in Proc. IEEE INFOCOM '96, Mar. 1996, vol. 3, pp. 1133-1140.

[4] L. Cheng and I. Marsic, "Modeling and prediction of session throughput of constant bit rate streams in wireless data networks," in Proc. IEEE WCNC 2003, Mar. 2003, vol. 3, pp. 1733-1741.

[5] S. Y. Cheung and C. S. Pencea, "BSFQ: Bin sort fair queueing," in Proc. IEEE INFOCOM 2002, Jun. 2002, vol. 3, pp. 1640-1649.

[6] R. Cusani, F. D. Priscoli, G. Ferrari, and M. Torregiani, "A novel MAC and scheduling strategy to guarantee QoS for the new generation WIND-FLEX wireless LAN," IEEE Wireless Commun. Mag., vol. 9, no. 3, pp. 46-56, Jun. 2002.

[7] P. Drews, D. Sommer, R. Chandler, and T. Smith, "Managed runtime environments for next-generation mobile devices," Intel Technol. J., vol. 7, no. 1, pp. 77-82, Feb. 2003.

[8] A. El Gamal, C. Nair, B. Parabhakar, E. Uysal-Biyiklioglu, and S. Zahedi, "Energy-efficient scheduling of packet transmissions over wireless networks," in Proc. IEEE INFOCOM 2002, Jun. 2002, vol. 3, pp. $1773-1782$.

[9] G. Holland, N. Vaidya, and P. Bahl, "A rate-adaptive MAC protocol for multi-hop wireless networks," in Proc. ACM MOBICOM '01, Aug. 2001, pp. 236-251.

[10] V. Huang and W. Zhuang, "Optimal resource management in packetswitching TDD CDMA systems," IEEE Pers. Commun., vol. 7, no. 6, pp. 26-31, Dec. 2000.

[11] V. Huang and W. Zhuang, "QoS-oriented access control for 4G multimedia CDMA communications," IEEE Commun. Mag., vol. 20, no. 3, pp. 118-125, Mar. 2002. 
[12] Wireless LAN Medium Access Control (MAC) and Physical Layer (phy) Specifications, IEEE 802.11, 1999.

[13] "The Intel(R) XScale(TM) Microarchitecture Technical Summary," Intel [Online]. Available: http://www.intel.com/design/intelxscale/

[14] D. N. Knisely, S. Kumar, S. Laha, and S. Nanda, "Evolution of wireless data services: IS-95 to cdma2000," IEEE Commun. Mag., vol. 36, no. 10, pp. $140-149$, Oct. 1998

[15] J. W. Lee, R. R. Mazumdar, and N. B. Shroff, "Downlink power allocation for multi-class CDMA wireless networks," in Proc. IEEE INFOCOM 2002, Jun. 2002, vol. 3, pp. 1480-1489.

[16] S. Lu, V. Bharghavan, and R. Srikant, "Fair scheduling in wireless packet networks," IEEE/ACM Trans. Netw., vol. 7, no. 4, pp. 473-489, Aug. 1999.

[17] Z. Naor and H. Levy, "A centralized dynamic access probability protocol for next generation wireless networks," in Proc. IEEE INFOCOM 2001, Apr. 2001, vol. 2, pp. 767-775.

[18] T. T. Nielsen and J. Wigard, Performance Enhancements in a Frequency Hopping GSM Network. Boston, MA: Kluwer Academic, 2000.

[19] S. Ramanathan, "A unified framework and algorithm for (T/F/C)DMA channel assignment in wireless networks," in Proc. IEEE INFOCOM '97, Apr. 1997, vol. 2, pp. 900-907.

[20] B. Sadeghi, V. Kanodia, A. Sabharwal, and E. Knightly, "Opportunistic media access for multirate ad hoc networks," presented at the ACM MOBICOM '02, Atlanta, GA, Sep. 2002.

[21] O. Sallent, J. P. Romero, R. Agusti, and F. Casadevall, "Provisioning multimedia wireless networks for better QoS: RRM strategies for 3G W-CDMA," IEEE Commun. Mag., vol. 41, no. 2, pp. 100-106, Feb. 2003.

[22] The Connected Limited Device Configuration (CLDC) HotSpot Implementation Virtual Machine (JAVA 2 Platform, Micro Edition). White Paper. Sun Microsystems [Online]. Available: http://www.java.sun.com/products/cldc/

[23] A. J. Viterbi, CDMA: Principles of Spread Spectrum Communication, 1st ed. Upper Saddle River, NJ: Prentice Hall PTR, 1995.

[24] X. Wang, "An FDD wideband CDMA MAC protocol for wireless multimedia networks," in Proc. IEEE INFOCOM 2003, Mar. 2003, vol. 1, pp. 734-744.

[25] C.-H. Yeh, "ACENET: Architectures and protocols for high throughput, low power, and QoS provisioning in next-generation mobile communications," in Proc. IEEE PIMRC 2002, Sep. 2002, vol. 2, pp. 779-786.

[26] C.-H. Yeh, "A TCDMA protocol for next-generation wireless cellular networks with bursty traffic and diverse QoS requirements," in Proc. IEEE PIMRC 2002, Sep. 2002, vol. 5, pp. 2142-2147.

[27] X. Zhu et al., "The RF module design for W-CDMA/GSM dual band and dual mode handset," in IEEE MTT-S 2001 Int. Microwave Symp. Dig., May 2001, vol. 3, pp. 2215-2218.

[28] Universal Mobile Telecommunications System (UMTS); Physical Channels and Mapping of Transport Channels Onto Physical Channels (FDD) 3GPP TS 25.211, version 5.2.0, release 5, Sep. 2002.

[29] Digital Cellular Telecommunications System (Phase 2+); Multiplexing and Multiple Access on the Radio Path 3GPP TS 05.02, version 8.8.0, Jan. 2001.

[30] Medium Access Control (MAC) Standard for cdma2000 Spread Spectrum Systems 3GPP2 C.S0003-0-2, version 1.0, release 0, addendum 2, Apr. 2001.

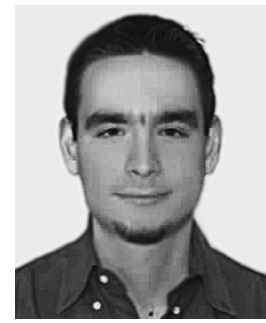

Mehmet C. Vuran (M'98) received the B.Sc. degree in electrical and electronics engineering from Bilkent University, Ankara, Turkey, in 2002, and the M.S. degree in electrical and computer engineering from the Georgia Institute of Technology, Atlanta, in 2004. He is currently a Research Assistant in the Broadband and Wireless Networking Laboratory and pursuing the Ph.D. degree in the School of Electrical and Computer Engineering, Georgia Institute of Technology, under the guidance of Prof. Ian F. Akyildiz.

He received the 2006 BWN Laboratory Researcher of the Year Award. His current research interests are in wireless sensor networks, cognitive radio networks, and deep space communication networks.

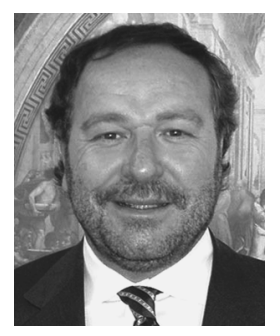

Ian F. Akyildiz (M'86-SM'89-F'96) received the B.S., M.S., and Ph.D. degrees in computer engineering from the University of Erlangen-Nuernberg, Germany, in 1978, 1981, and 1984, respectively.

Currently, he is the Ken Byers Distinguished Chair Professor with the School of Electrical and Computer Engineering, Georgia Institute of Technology, Atlanta, and Director of Broadband and Wireless Networking Laboratory. He is an Editor-in-Chief of the Computer Networks (Elsevier) and of the AdHoc Network Journal (Elsevier). His current research interests are in sensor networks, wireless mesh networks, and cognitive radio networks.

Dr. Akyildiz received the Don Federico Santa Maria Medal for his services to the Universidad of Federico Santa Maria in 1986. From 1989 to 1998, he served as a National Lecturer for ACM and received the ACM Outstanding Distinguished Lecturer Award in 1994. He received the 1997 IEEE Leonard G. Abraham Prize Award (IEEE Communications Society) for his paper entitled "Multimedia Group Synchronization Protocols for Integrated Services Architectures" published in the IEEE JouRnAl OF SELECTED AREAS IN COMMUNICATIONS (JSAC) in January 1996. He received the 2002 IEEE Harry M. Goode Memorial Award (IEEE Computer Society) with the citation "for significant and pioneering contributions to advanced architectures and protocols for wireless and satellite networking". He received the 2003 IEEE Best Tutorial Award (IEEE Communication Society) for his paper entitled "A Survey on Sensor Networks," published in IEEE Communications Magazine, in August 2002. He also received the 2003 ACM Sigmobile Outstanding Contribution Award with the citation "for pioneering contributions in the area of mobility and resource management for wireless communication networks." He received the 2004 Georgia Tech Faculty Research Author Award for his outstanding record of publications of papers between 1999-2003, April 2004 $\mathrm{He}$ also received the 2005 Distinguished Faculty Achievement Award from the School of ECE, Georgia Tech, April 2005. He has been a Fellow of the Association for Computing Machinery (ACM) since 1996. 